\title{
Research Paper \\ Effects of 8 Weeks Aerobic Training on Plasma Ghrelin Level and Ghrelin Lymphocyte Gene Expression in Elderly Men
}

\author{
Seyyed Mahdi Ahmadi ${ }^{1}$, ${ }^{*}$ Mehrdad Fathi ${ }^{1}$, Amir RashidLamir ${ }^{1}$, Farnaz Aminian ${ }^{2}$
}

1. Department of Physiology, Faculty of Physical Education and Sports Sciences, Ferdowsi University of Mashhad, Mashhad, Iran. 2. Department of Physiology, Faculty of Humanities, Bojnourd Branch, Islamic Azad University, Bojnourd, Iran.

\begin{tabular}{|c|c|}
\hline $\begin{array}{l}\text { Use your device to scan } \\
\text { and read the article online }\end{array}$ & 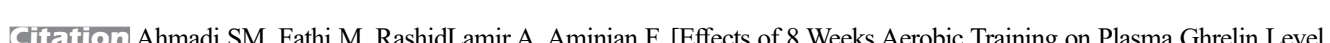 \\
\hline 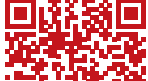 & $\begin{array}{l}\text { and Ghrelin Lymphocyte Gene Expression in Elderly Men (Persian)]. Iranian Journal of Ageing. 2019; 13(4):494-505. https://doi. } \\
\text { org/10.32598/SIJA.13.4.494 }\end{array}$ \\
\hline 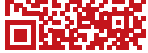 & d cil" https://doi.org/10.32598/SIJA.13.4.494 \\
\hline
\end{tabular}

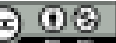

Received: 13 Jun 2018 Accepted: 10 Nov 2018 Available Online: 01 Jan 2019

Keywords: Aerobic training Ghrelin, Elderly men

\section{A B STRACT}

Objectives Ghrelin is a peptide hormone secreted from the endocrine cells of stomach, affecting feeding behavior, appetite, energy consumption, and body weight. The present study compared the effect of 8 weeks of aerobic training on plasma ghrelin and lymphocyte ghrelin gene expression in elderly men. Methods \& Materials In this quasi-experimental study, 30 subjects were selected by convenience sampling method. They were randomly divided into 2 equal groups of aerobic training $(n=15)$ and control $(n=15)$. Aerobic training program included aerobic trainings up to $50-60$ minutes at $60-85 \%$ of the maximum heart rate, 4 sessions per week which lasted for 8 weeks. After initial assessments (body composition and ghrelin gene expression), followed by 8 weeks of exercise training, post-intervention assessments were completed 48 hours after the last training session. To make intra- and between-group comparisons, repeated measures Analysis of Variance (ANOVA) was used. For all statistical comparisons, the level of significance was set at $\mathrm{P}<0.05$.

Results The Mean $\pm S D$ age ranges of the study participants were $69.13 \pm 3.68$ years in the training group and $69.20 \pm 4.31$ in the control group. Eight weeks of aerobic training significantly reduced BMI from 27.57 to $26.90 \mathrm{~kg} / \mathrm{m}^{2} \quad(P=0.001)$, and body fat percentage from $24.31 \%$ to $21.43 \%(P=0.001)$ in the training group. In addition, lymphocyte ghrelin gene expression significantly increased from $16.87 \%$ to $24.92 \%$ in the aerobic training group. There was no significant changes in plasma ghrelin levels.

Conclusion Aerobic training through increasing the lymphocyte ghrelin gene expression level and decreasing the values of BMI in middle-aged men, can prevent the incidence of obesity and overweight. It is reasonable to support the role of physical activity as a non-prescriptive therapy in regulating balance and effective peptide hormones in the prevalence of obesity. Therefore, aerobic exercises can be used as a preventive method for the reduction of obesity and the incidence of related diseases.

\section{Extended Abstract}

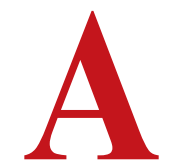

\section{Objectives}

ppetite is among the most influential factors in energy homeostasis and its regulation plays an important role in controlling energy balance [1]. The environmental factors involved in energy homeostasis and appetite regulation are often controlled by long-term signals such as ghrelin, cholecystokinin, and peptide YY [2]. Ghrelin, an acylated

\section{* Corresponding Author}

\section{Mehrdad Fathi, PhD.}

Address: Department of Physiology, Faculty of Physical Education and Sports Sciences, Ferdowsi University of Mashhad, Mashhad, Iran.

Tel: +98 (915) 2570058

E-mail: mfathei@um.ac.ir 
upper gastrointestinal peptide, is the only known orexigenic hormone [2]. According to studies, of 3 types of mutations in the preproghrelin gene, only 1 is associated with obesity in humans [3]. Thus, overweight and obesity and its related diseases have become a major economic problem in many countries due to their high health and medical costs. The positive effects of exercise and physical activity on preventing obesity and overweight have been reported [4]. The current study evaluated the effect of aerobic training on plasma ghrelin level and the expression of ghrelin lymphocyte gene in elderly men.

\section{Methods and Materials}

In this quasi-experimental study, 30 elderly men with an average age of 60-70 years and Body Mass Index (BIM) of $25-30 \mathrm{~kg} / \mathrm{m}^{2}$ participated. The subjects were selected using convenience and purposeful sampling methods. They were then randomly divided into 2 groups of training $(n=15)$ and control $(n=15)$. Those in the training group received aerobic training for 8 weeks; 4 sessions per week. The duration of each session was 50-60 minutes. The intervention program included: 1 . A 10-minute warmup; 2. Aerobic training for 50-60 minutes with an intensity of $60-85 \%$ of the maximum heart rate. The training time gradually increased from 20 minutes at the beginning to 45 minutes at the end; and 3. A 10-minute cool down. The intensity of training was measured by a heart rate sensor (Polar, England).
Blood samples were collected 48 hours before initiating the training and 24 hours after the last training session, to measure plasma ghrelin levels using ELISA method, and the expression of ghrelin lymphocyte gene using semiquantitative Reverse Transcription-Polymerase Chain Reaction (RT-PCR) analysis with the sequence of forward primer (5'-ATGTGGAACGCGACCCCCAGCGA-3') and reverse primer (5'-ACCCCCAATTGTTTCCAGACCCAT-3'). The obtained data were analyzed by SPSS. The between-group and within-group comparisons were conducted using repeated measures Analysis of Variance (ANOVA). The obtained results were evaluated at a significance level of $P<0.05$. This paper was extracted from a master thesis and has been approved by the Ethics Committee of the Faculty of Sport Sciences at Ferdowsi University of Mashhad (code: 18731).

\section{Results}

According to the obtained results, no significant differences were found between the 2 study groups in terms of height, BMI, body fat percentage, plasma ghrelin level and the expression of ghrelin lymphocyte gene. ANOVA results presented in Table 1, indicated that an 8 -week aerobic training with an intensity of $60-85 \%$ of the maximum heart rate could significantly reduce BMI from 27.57 to $26.90 \mathrm{~kg} / \mathrm{m}^{2}(\mathrm{P}=0.001)$ and body fat percentage from $24.31 \%$ to $21.43 \%(\mathrm{P}=0.001)$, and could significantly increase the expression of ghrelin lymphocyte gene from $16.87 \%$ to $24.92 \%$. Moreover,

Table 1. Between-group and within-group comparisons of changes in plasma ghrelin level and the expression of ghrelin lymphocyte gene

\begin{tabular}{|c|c|c|c|c|c|c|c|}
\hline \multirow{3}{*}{ Variables } & \multirow{3}{*}{ Groups } & \multicolumn{2}{|c|}{ Mean \pm SD } & \multicolumn{4}{|c|}{ Changes } \\
\hline & & \multirow{2}{*}{ Pre-Test } & \multirow{2}{*}{ Post-Test } & \multicolumn{2}{|c|}{ Within Group } & \multicolumn{2}{|c|}{ Between Group } \\
\hline & & & & $\mathbf{F}$ & $\mathbf{P}$ & $\mathbf{F}$ & $\mathbf{P}$ \\
\hline \multirow{2}{*}{$\mathrm{BMI}, \mathrm{kg} / \mathrm{m}^{2}$} & Training & $27.57 \pm 1.59$ & $26.58 \pm 1.90$ & 169.27 & $0.000^{*}$ & \multirow{2}{*}{0.12} & \multirow{2}{*}{0.73} \\
\hline & Control & $27.07 \pm 1.14$ & $27.13 \pm 1.05$ & 0.14 & 0.713 & & \\
\hline \multirow[b]{2}{*}{ Body fat, \% } & Training & $24.31 \pm 3.39$ & $21.43 \pm 2.94$ & 153.27 & $0.000^{*}$ & \multirow[b]{2}{*}{1.01} & \multirow[b]{2}{*}{0.32} \\
\hline & Control & $23.93 \pm 3.03$ & $24.06 \pm 2.96$ & 0.68 & 0.424 & & \\
\hline \multirow[b]{2}{*}{ Plasma ghrelin level, $\mathrm{ng} / \mathrm{mL}$} & Training & $0.64 \pm 0.11$ & $0.71 \pm 0.02$ & 3.30 & $0.002^{*}$ & \multirow[b]{2}{*}{6.77} & \multirow[b]{2}{*}{$0.01^{*}$} \\
\hline & Control & $0.57 \pm 0.11$ & $0.59 \pm 0.09$ & 0.79 & 0.396 & & \\
\hline \multirow{2}{*}{$\begin{array}{l}\text { Ghrelin lymphocyte gene expres- } \\
\text { sion, } \mathrm{ng} / \mathrm{mL}\end{array}$} & Training & $16.87 \pm 2.34$ & $24.92 \pm 8.62$ & 6.11 & 0.046 & \multirow{2}{*}{0.29} & \multirow{2}{*}{0.60} \\
\hline & Control & $19.38 \pm 1.64$ & $20.07 \pm 2.43$ & 2.34 & 0.187 & & \\
\hline
\end{tabular}

* Significant at $\mathrm{P}<0.05$ 
plasma ghrelin level increased from 0.64 to $0.71 \mathrm{ng} /$ $\mathrm{mL}$; however, this increase was not significant.

The Independent t-test results regarding betweengroup comparison of posttest mean scores suggested no significant differences between the 2 groups in terms of BMI, body fat percentage, and the expression of ghrelin lymphocyte gene $(\mathrm{P}>0.05)$ after the intervention. However, in terms of plasma ghrelin level, a significant difference was observed between the groups. In other words, aerobic training had a significant effect on the increase of plasma ghrelin level in the elderly men.

\section{Conclusion}

An 8-week aerobic exercise resulted in a significant decrease in body fat percentage and BMI in the training group. However, the expression of ghrelin lymphocyte gene significantly increased. No significant difference was observed in plasma ghrelin level after the intervention. Therefore, regular aerobic exercise can be used as a non-pharmacological treatment in regulating the balance of peptide hormones, effective in the prevalence of obesity. Possibly, the aerobic exercise causes a negative energy balance in the body. In response to energy shortages, ghrelin stimulates food intake, resupplies lost energy resources, and restores energy balance to compensate for energy reserves in the body. Further studies are required for the recognition of main mechanisms responsible for the association of ghrelin with obesity and the role of physical activity on them.

\section{Ethical Considerations}

\section{Compliance with ethical guidelines}

All ethical principles were considered in this article. The participants were informed about the purpose of the research and its implementation stages; they were also assured about the confidentiality of their information; Moreover, They were allowed to leave the study whenever they wish, and if desired, the results of the research would be available to them. This paper has been approved by the Ethics Committee of the Faculty of Sport Sciences at Ferdowsi University of Mashhad (code: 18731).

\section{Funding}

The present paper was extracted from the MSc. thesis of the first author, in Department of Physiology, Fac- ulty of Physical Education and Sports Sciences, Ferdowsi University of Mashhad.

\section{Authors contributions}

All authors contributed in preparing this article.

\section{Conflict of interest}

The authors declared no conflict of interest. 


\title{
تأثير هشت هفته تمرين هوازى بر سطح ترلين يلاسما و بيان زٔن ترلين لنفوسيت مردان سالمند
}

\author{
سيدمهدى احمدى'، • مهرداد فتحى'، امير رشيد لمير' •، فرناز امينيان' \\ 1- كروه فيزيولوزى ورزشى، انشكده علوم ورزشى، داتشكاه فردوسى مشهيد، مشهيد، ايران.

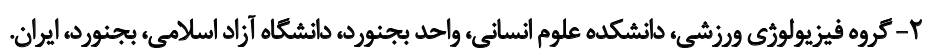

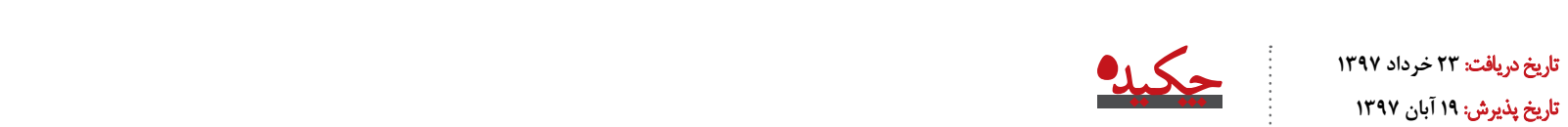

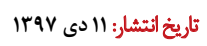

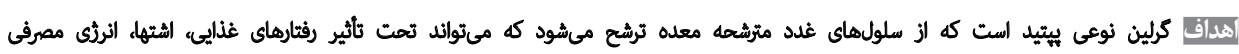

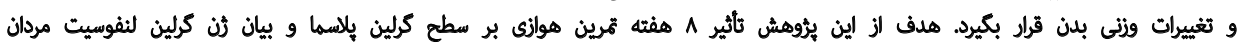
سالمثدبود.

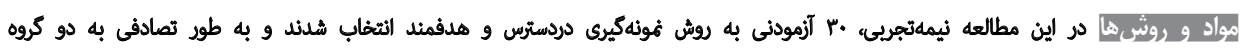

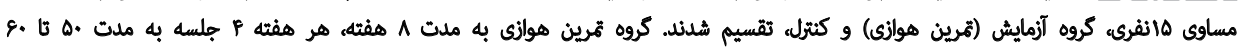

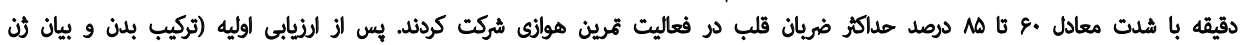

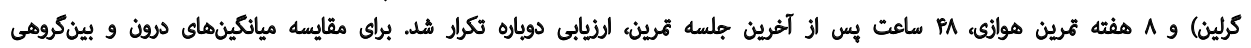

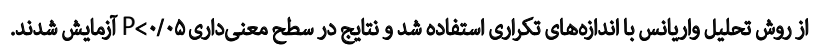

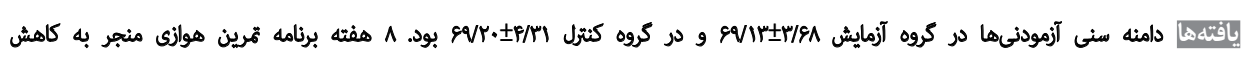

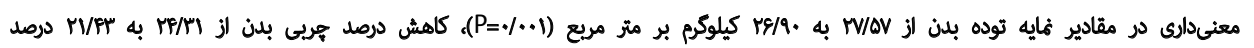

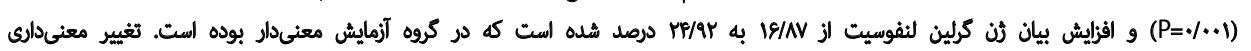
در سطح كرلين بلاسما ديده نشد.

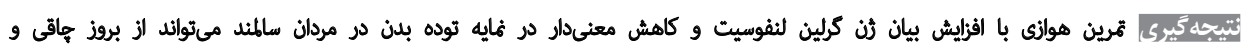

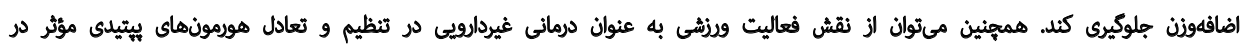

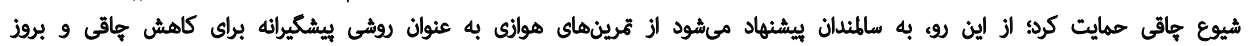
بيهماريهاى مرتبط با آن استفاده كنثل.

اسيدآمينه است؛ "س اسيدآمينه از توالى سيكنال، وع اسيدآمينه

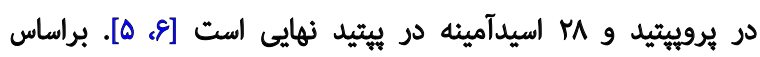

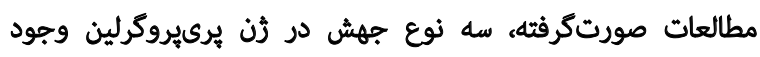

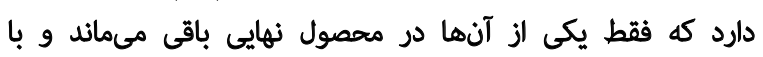

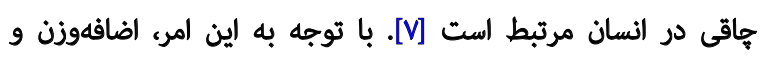

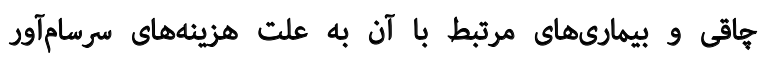

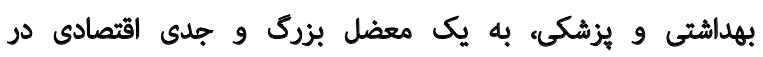
بسيارى از كشورها تبديل شده است. به يك

از طرفى تعادل منفى انرذى نيز باعث بروز اختلالاتى از جمله

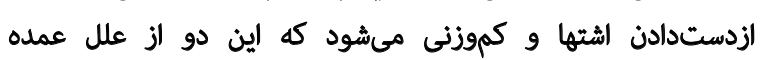

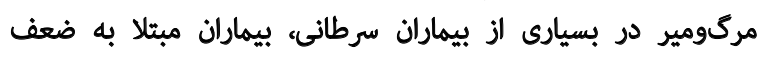

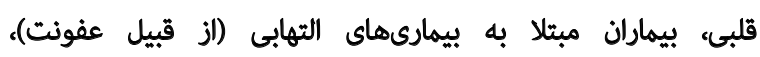

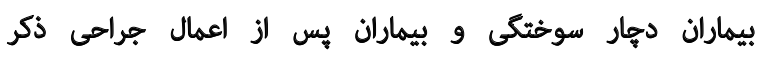

مقدمه

اشتها از موارد تأثيركذار بر هومئوستاز انرثى است و تنظيم أنقار

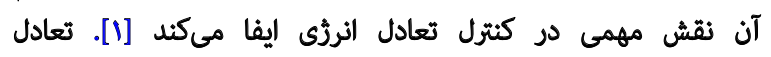

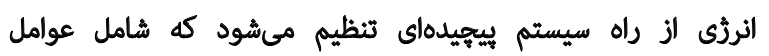

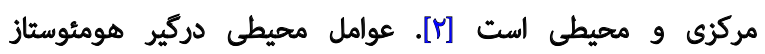

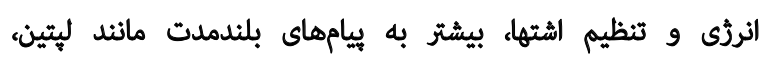

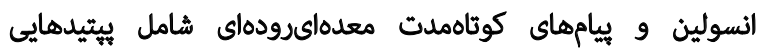

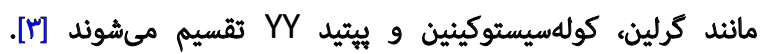

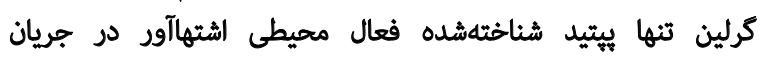

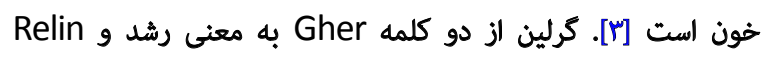

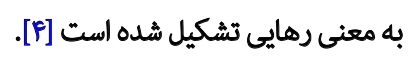

IIV يركيروكرلين در انسان از F اكزورن كد مىشود كه شامل 
آمارى اين تهقيق را مردان غيرفعال ساكن شهرستان نيشابور با

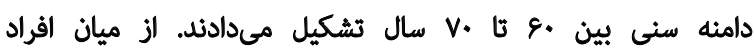

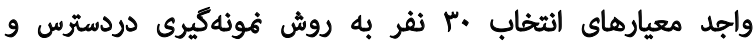

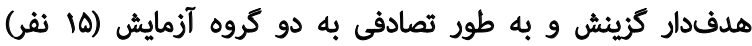

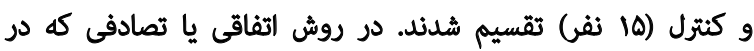

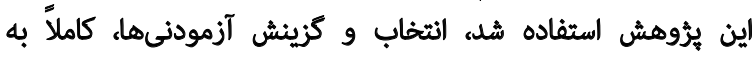

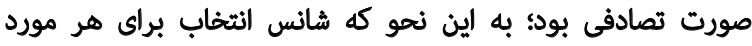

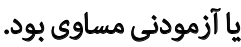

در اين روش از مدل تصادفىسازى ساده مثل قرعهكشى

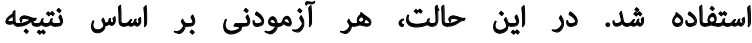

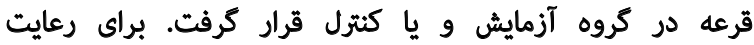

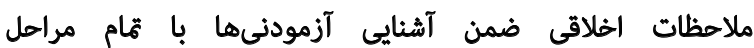

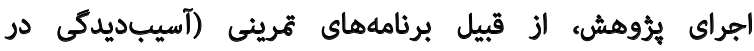

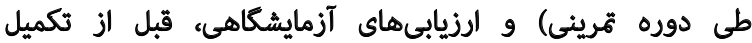

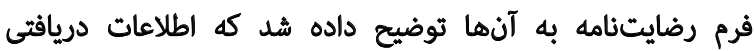

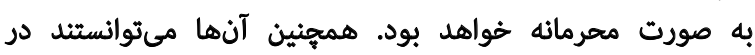
صورت تمايل در هر مرحلهائ از مطالعه خارج شوند.

در مرحله نخست افراد با ماهيت و نحوه همكارى براى اجراى

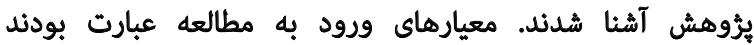

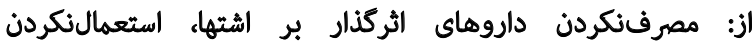

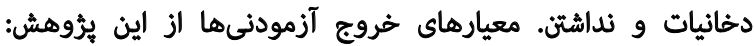

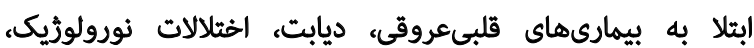

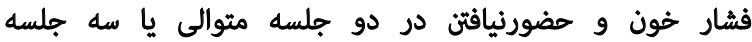

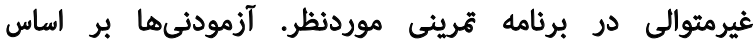

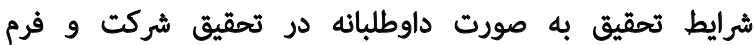
رضايتنامه را امضا كردند.

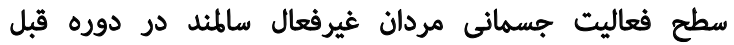

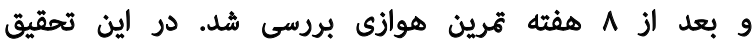

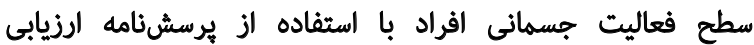

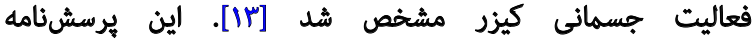

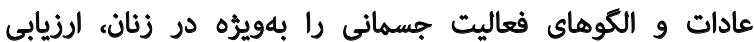

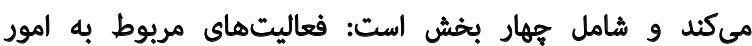

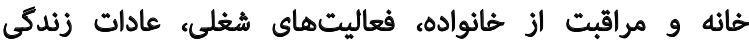

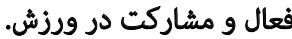

استرن فرلد، آينس ورث وكوسنبرى' در سال (1999) اين ابزار

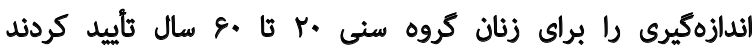

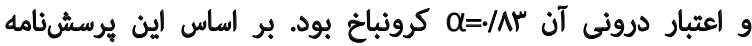

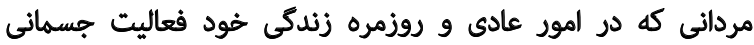

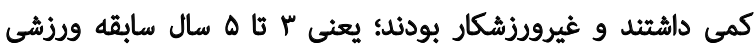

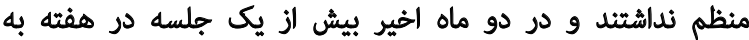

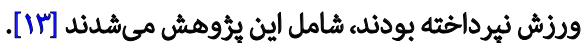

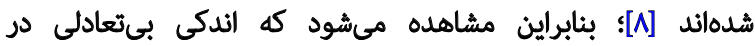

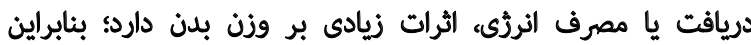

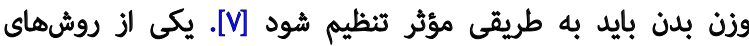
اثركذار بر وزن بدن، انجام فعاليتهاى بدنى منظم است.

فعاليت بدنى عاملى اصلاجيذير در سبك زندكى است كه

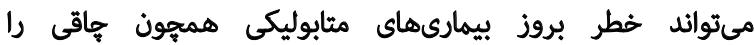

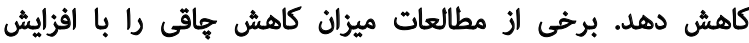

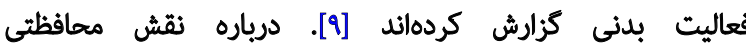

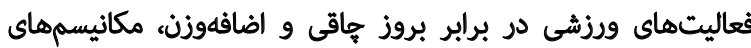

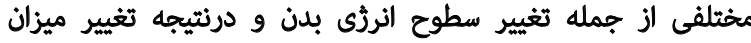

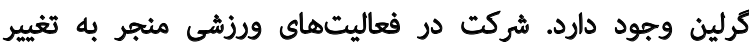

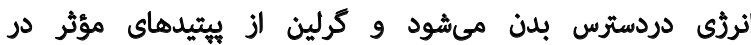

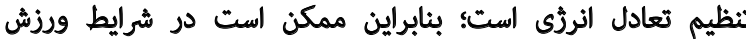
دستخوش تغييرات شود [•1].

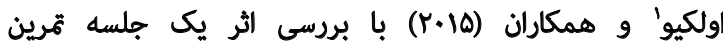

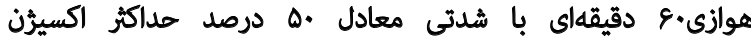

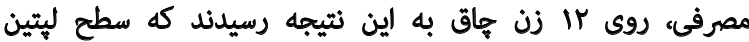

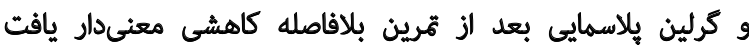

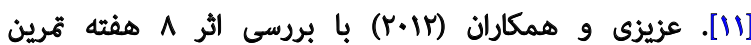

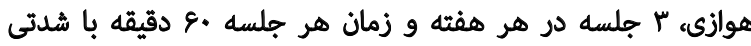

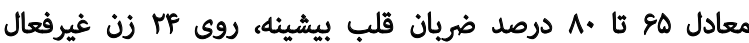

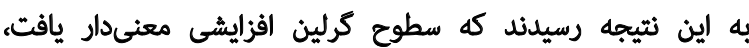

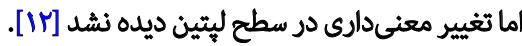

در نتيجه، با توجه به اينكه فعاليت بدنى متغيرترين بخش الز

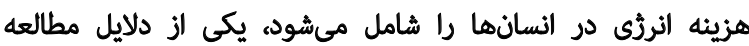

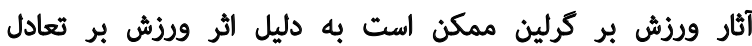

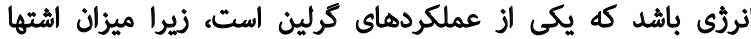

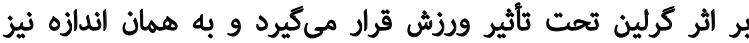

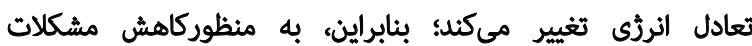

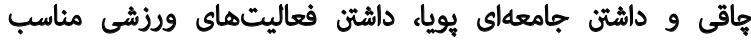

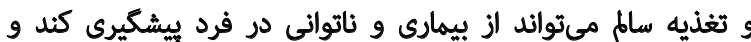

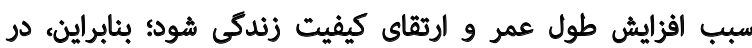

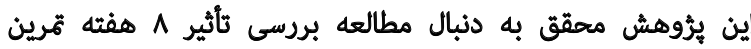

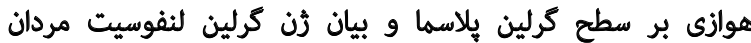

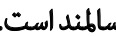

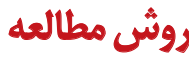$$
\text { أزمودنىها }
$$

اين تحقيق از نوع نيمهتجربى است كه با طرح دو كروه آزمايش و كنترل با ييشآزمون و بيسآزمون انجام شد. نهونه 
ثمونهكيرى در بين ساعات ^ منا أصبع در آزمايشكاه از سياهرى

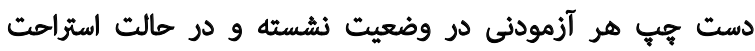

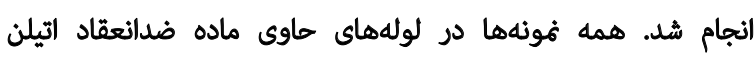

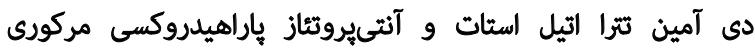
بنزوئيك اسيد قرار كرفت (يك ميلى مولار در حجم نهايى نمونه).

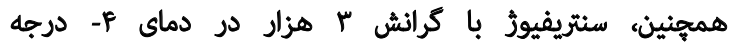

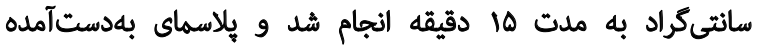

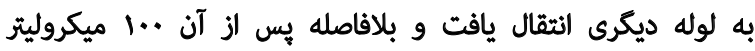

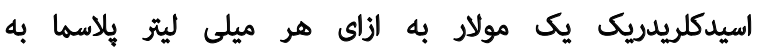

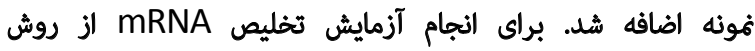

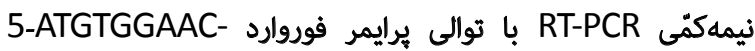
5-ACCCCCAATT- و يرايمر ريورس فورود GCGACCCCCAGCGA-3

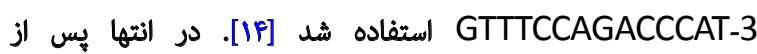
Gel Doc-It مدل بلدستآمدن نتايج با استفاده از دستكاه ساخت كشور آمريكا و بهدست آوردن مقدار بتاكتين،

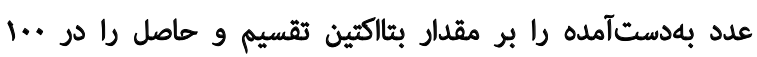

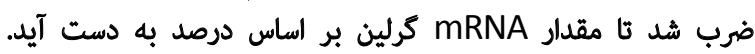

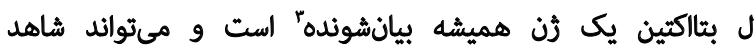

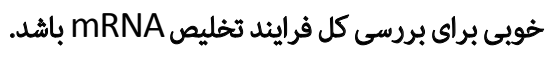

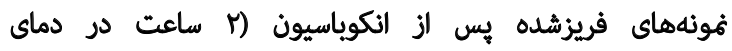

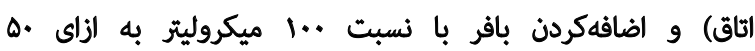
ميكروليتر نهونه و r بار شستوشو بافيت بان روش الايزا و و با استفاده

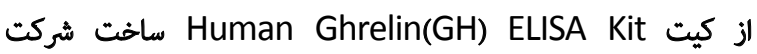
كشور جين و تحت ليسانس آمريكا آزمايش شرك

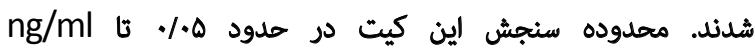

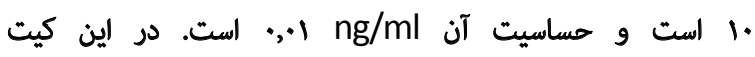

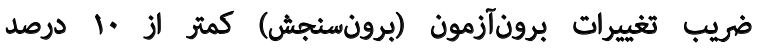

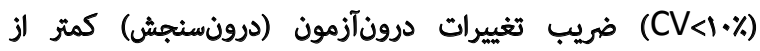
rا

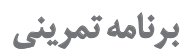

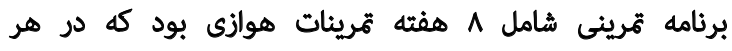

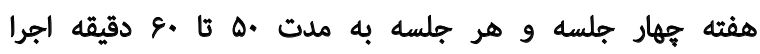

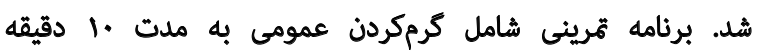

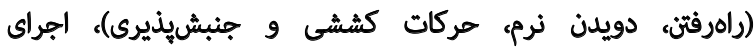

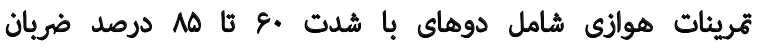

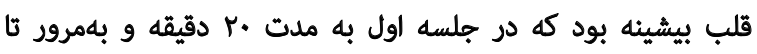
باياين ^ هفته به •و دقيقه افزايش يافت.

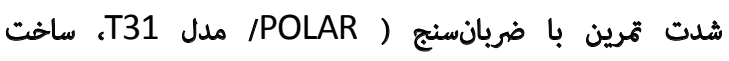

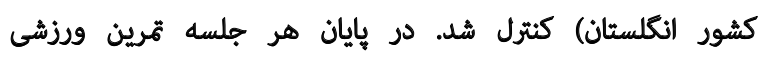
به مدت •1 دقيقه بازگتت بدن به حالت الت اوليه و سردكردن

3. House keeping gene

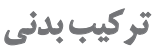

براى ارزيابى تركيب بدنى طول قد آزمودنىها از قدسنج سكا

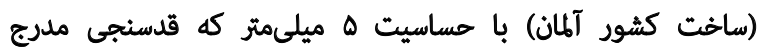

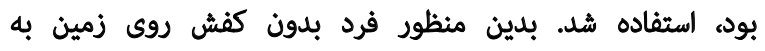

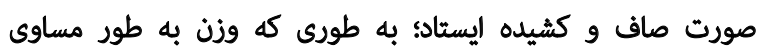

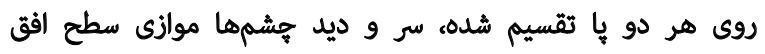

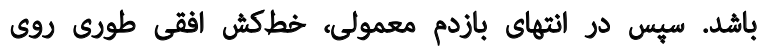

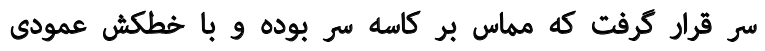

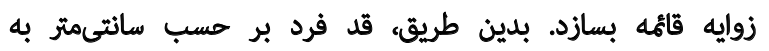

$$
\text { دست آمد. }
$$

وزن آزمودنىهاى واجد شرايط با ترازوى ديجيتالى كميانى Beurer

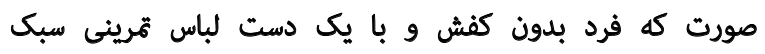

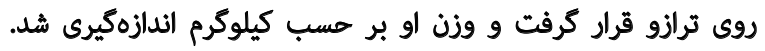

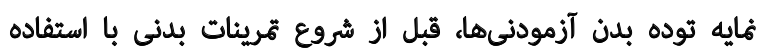

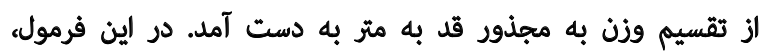

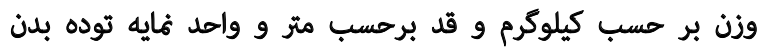
بر حسب كيلوكرم متر مربع است.

سيس براي اندازهكيرى درصد هربى بدن با استفاده از كاليير

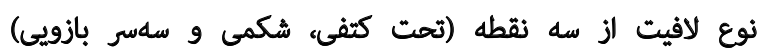

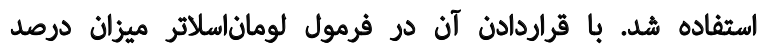

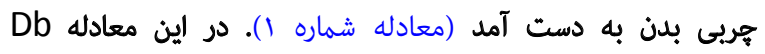

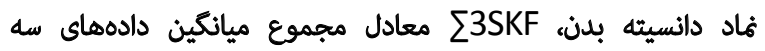
نقطه الدازمكيرى نده و BF\% هاد درصد هيربى بدن است.

معادله شماره ا:

\section{$D b=\left[1.0973-\left(0.000815 \times \sum 3 S K F\right)\right]+\left[0.00000084 \times\left(\sum\right.\right.$} $\left.3 S K F)^{2}\right]$

\section{$B F \%=(457.0 \times D b)-414.2$}

آزمودنىها يَس از معاينه قلبىعروقى، اندازمكيرى فشارخون

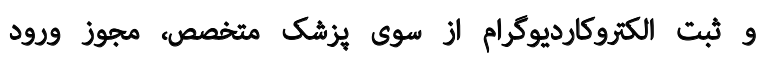

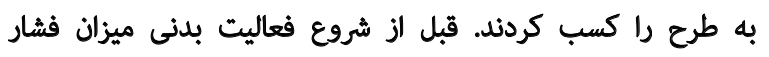

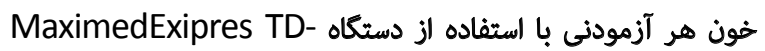

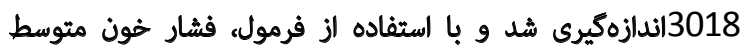

شريانى به فشار خون متوسط تبديل شد (معادله شمارهب).

معادله شمارهr: - (ب)

"r/(فشار خونسيتولى+فشار خوندياستولى×|)=فشارخونمتوسط

$$
\text { نمونه ئيرى خونى }
$$

در اين تحقيق ثمونههاي خونى در FA ساعت بيش از شروع

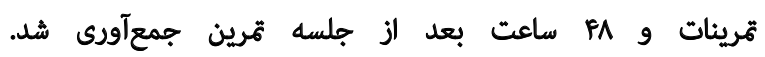


F جلسه و هر جلسه به مدت .ه ثا .9 دقيقه با شدتى معادل

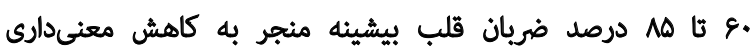

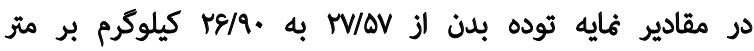

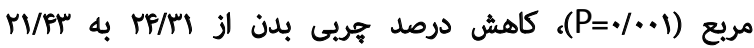

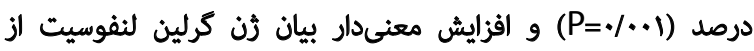
I I ب/AV

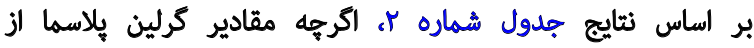

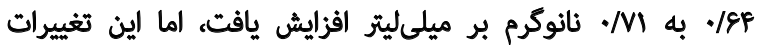

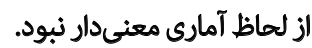

تغييرات ميانكينهاى بينكروهى در مثغيرهاى شاخص توده

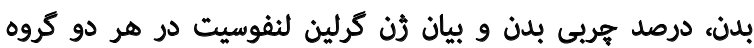

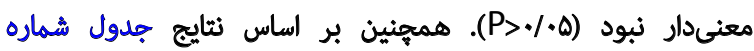

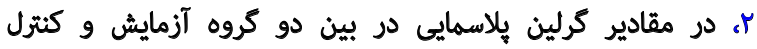

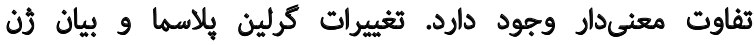

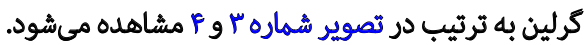

$\Leftrightarrow$

براساس ثتايج بهدستآمده از اين تحقيق، $\wedge$ هفته برثامه ثمرين

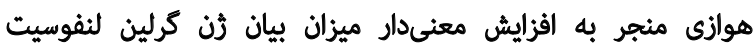

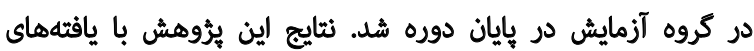

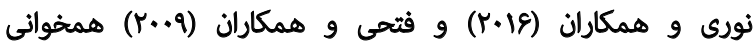

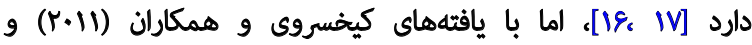

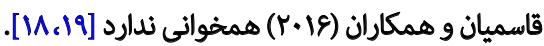

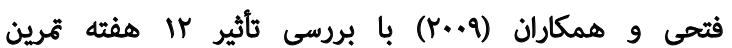

(دويدن آهسته، راهرفني و حركات كششى) النجام مى مأشد [10].

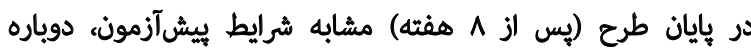

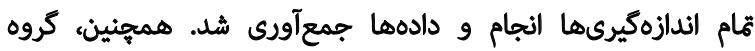

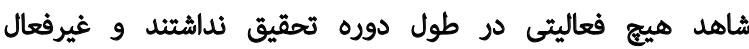
بودند (همجون قبل از اين مطالعه، شيوه زندكى غير فئنال داشتئد).

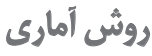

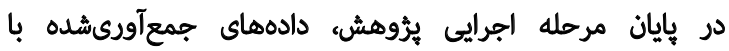

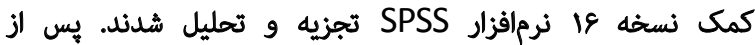
تأييد نرمالبودن توزيع نظرى دادهها با استفاده از آزمون آماريه

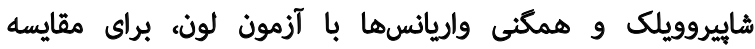

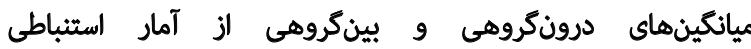

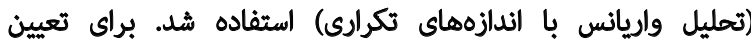
معنى در نظر كرفته شد.

Ldị̂ls

مشخصات آزمودنىهاى كروه آزمايش و كنترل در جدول شماره

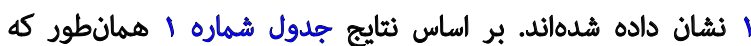
مشاهده مىشود، تفاوت معنىدارى بين شاخصهاي قد، درصد

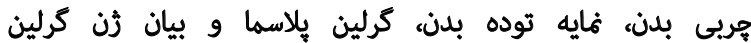

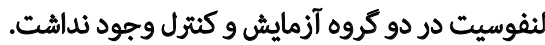
بر اساس جدول شماره ب ب، نتايج تهليل والريانس با اندازههاى

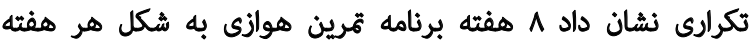

جدول ا. ميانكين، انحراف معيار و همجنين نتايج آزمون تى مستقل براى بررسى فرض همكنبودن واريانسها در شروع دوره تمرين

\begin{tabular}{|c|c|c|c|c|}
\hline \multicolumn{2}{|c|}{ آزمون لون براى تعيين برابرى واريانسها } & \multirow{2}{*}{ ميانثين |اثحر اف معيار } & \multirow{2}{*}{$\log$} & \multirow{2}{*}{ اندازههاي تن سنجمى } \\
\hline$P$ & $F$ & & & \\
\hline \multirow{2}{*}{+} & \multirow{2}{*}{ - } & $\mid W / \& r \pm r / q \Delta$ & 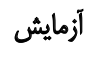 & قد \\
\hline & & $I V \Delta / V E \pm F / F q$ & كنترل & (سانتىمتر) \\
\hline \multirow{2}{*}{.$|1|$} & \multirow{2}{*}{$+1+\Delta$} & $r e / r I \pm r / r q$ & 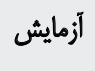 & \multirow{2}{*}{ درصل جريى بلن } \\
\hline & & $r / q \mu \pm r / . r$ & 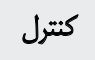 & \\
\hline \multirow{2}{*}{$.1 \cdot 1$} & \multirow{2}{*}{$r / 18$} & $r V / \Delta V \pm V / \Delta q$ & 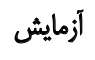 & \multirow{2}{*}{ (كيلوكرم/ متر مربع بلن } \\
\hline & & $r V / \cdot V \pm V / / F$ & كنترل & \\
\hline \multirow{2}{*}{.$/ 91$} & \multirow{2}{*}{$.1 . .1$} & $+|9+ \pm+| M \mid$ & أزمايش & \multirow{2}{*}{ 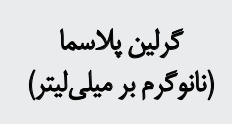 } \\
\hline & & $\cdot / \Delta V \pm+/ 11$ & 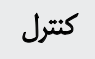 & \\
\hline \multirow[t]{2}{*}{. $\mid 81$} & \multirow{2}{*}{.$/ K \varepsilon$} & $\mid \varepsilon / \wedge V \pm r / M F$ & 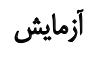 & \multirow{2}{*}{ بيان ثن كرلين الثفوسيت } \\
\hline & & 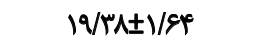 & كنترل & \\
\hline
\end{tabular}

212 
جدول r. مقايسه تغييرات واريانس درون كروهى و بين كروهى در سطح كرلين يلاسما و بيان رُن گرلين لثفوسيت مردان سالمثد

\begin{tabular}{|c|c|c|c|c|c|c|c|}
\hline \multicolumn{4}{|c|}{ تغييرات } & \multicolumn{2}{|c|}{ ميانكين土|نحراف معيار } & \multirow{3}{*}{ كروهها } & \multirow{3}{*}{ هتغيرها } \\
\hline \multicolumn{2}{|c|}{ بين كروه } & \multicolumn{2}{|c|}{ دوون } & \multirow{2}{*}{ يس آزمون } & \multirow{2}{*}{ ييش أزمون } & & \\
\hline $\mathbf{F}$ & $\mathbf{P}$ & $\mathbf{F}$ & $\mathbf{P}$ & & & & \\
\hline \multirow{2}{*}{$\cdot M$} & \multirow{2}{*}{$+/ M$} & $.1 \ldots *$ & $1 E q / T r$ & $r \& / \Delta \Lambda \pm 1 / A$. & $r V / \Delta V \pm V / \Delta q$ & أزمايش & \multirow{2}{*}{ (كيلوكرم بر مثر مربع) } \\
\hline & & .MIr &.$/ N$ & $\| V /|r \pm\rangle / \cdot \Delta$ & $Y V / \cdot Y \pm V / / F$ & كنترل & \\
\hline \multirow{2}{*}{.$/ M$} & \multirow{2}{*}{$1 / \cdot 1$} & $+1 \ldots *$ & $1 \Delta T / T Y$ & $r / / F t+r / a F$ & $r F / r \mid \pm r / r q q$ & أزمايش & \multirow{2}{*}{ درصد جربى بلن } \\
\hline & & . /RTF &.$/ 8 A$ & $r f / . \& \pm r / q$ & $r / q \Psi+r / \%$ & كنترل & \\
\hline \multirow{2}{*}{$+1+1^{*}$} & \multirow{2}{*}{$g / W$} & $+*+r^{*}$ & $r / r+$ & $\cdot M I \pm \cdot / \cdot r$ & $\cdot|x+ \pm \pm / 1|$ & أزمايش & \multirow{2}{*}{ (نانوكرم بر ميلىليتي) } \\
\hline & & $+/$ ras & $\cdot / N 9$ & $. / \Delta 9 \pm+1.9$ & $\cdot / \Delta v \pm+/ 11$ & كنترل & \\
\hline \multirow{2}{*}{.18} & \multirow{2}{*}{.$/ 7 q$} & $1+1+8^{*}$ & $9 / 11$ & TF/ATINGT & $\mid g / A V \pm r / M f$ & أزمايش & \multirow{2}{*}{ بيان ثن كرلين لنفوسيت } \\
\hline & &.$/ M A Y$ & $r / M$ & $r \cdot 1 \cdot V \pm T / T^{e}$ & $19 / \mathrm{r} A \pm 1 / 9 \mathrm{~A}$ & كتترل & \\
\hline
\end{tabular}

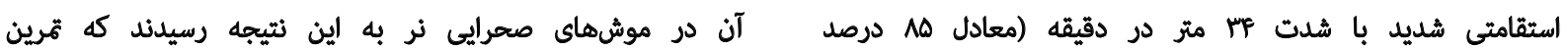

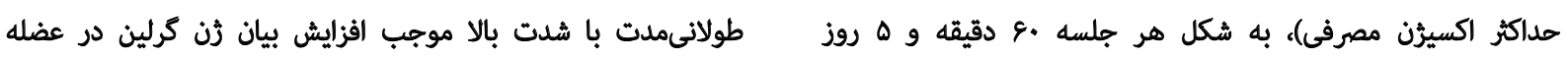

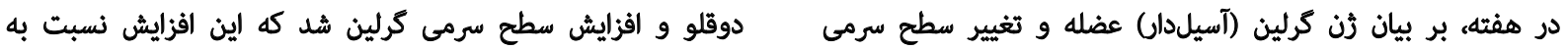
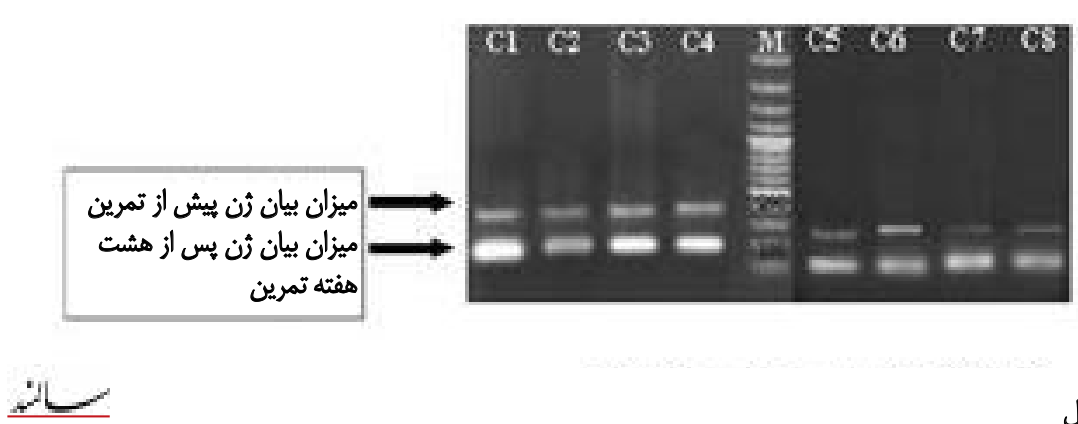

ميزان بيان ثن ييش از تهرين

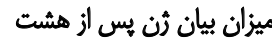

تصوير ا. الكتروفورز از بيان ثن كرلين در كروه كنترل C1 C1 C1 بيانكر افراد تستشده است.

هفته تمرين

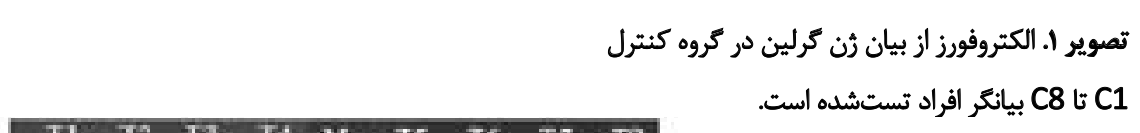

II T2 I3 I4 $\mathrm{M}$ IS T6 T7 TS

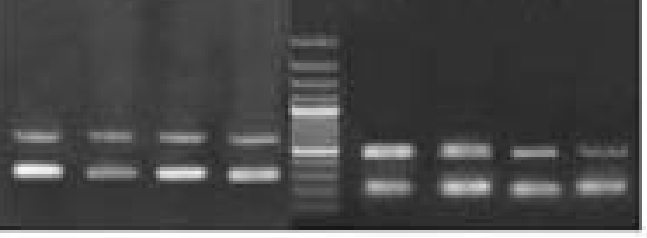

اين دو شكل بيان رن بتاكتين را نشان ميدها

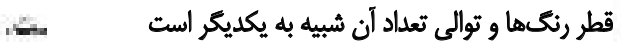

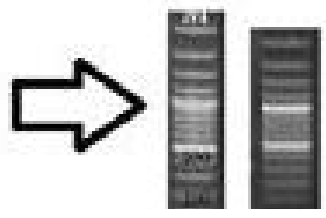

تصوير r. الكتروفورز از بيان ثن كرلين در كروه تمرين

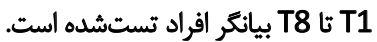




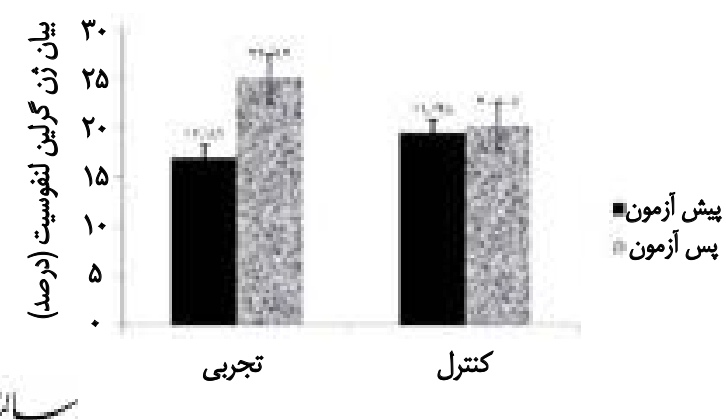

تصوير F. تغييرات سطع بيان رُن كرالين لنفوسيت در دو كروه آزمايش و كنترل

هنين تغييرى مشاهده نشده است؛ بنابراين، اين مطالعه براى

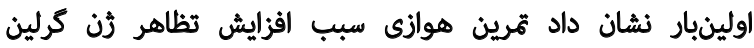

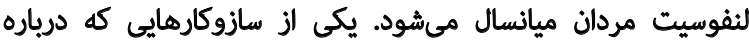

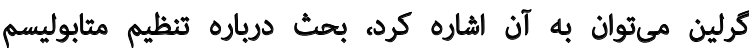

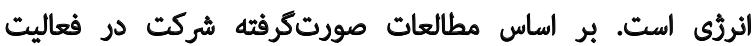

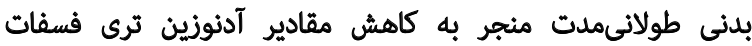

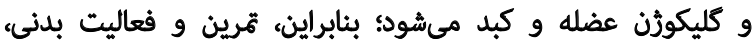

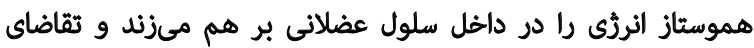

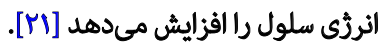

بروز حالت تعادل منفى انررى مىتواند ناشى از كاهش مقادير

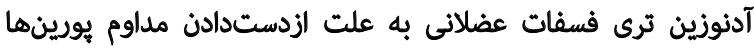

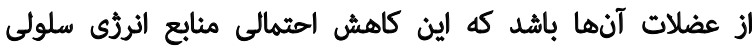

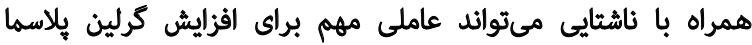

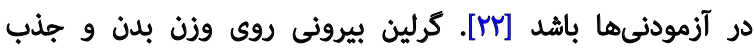

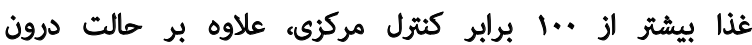

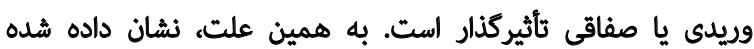

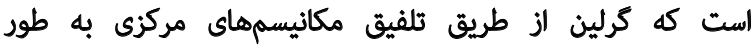
قابلتوجهى روى هوموستاز انرزى تأثير مى كذارد [بr]].

در هييوثالاموس، كرلين، تأثيراتش را به صورت مستقل با انتشار

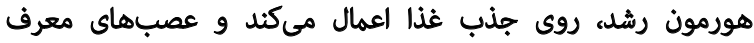
GHS-R

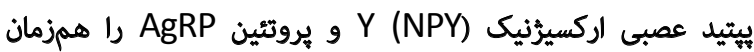

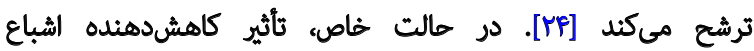

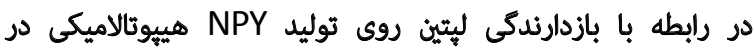

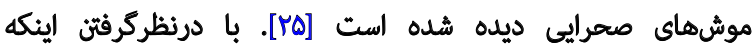

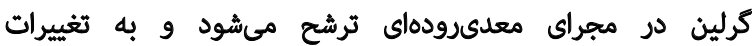

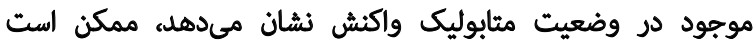

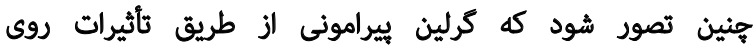

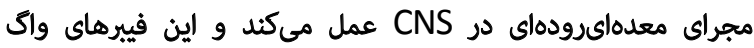

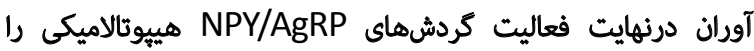
از طريق رله مغز يسين تغيير ميدهند.

نقش مهم عصب واك آوران به عنوان محرك رفتار تغذيه

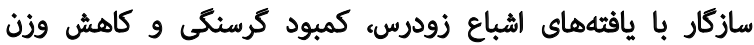
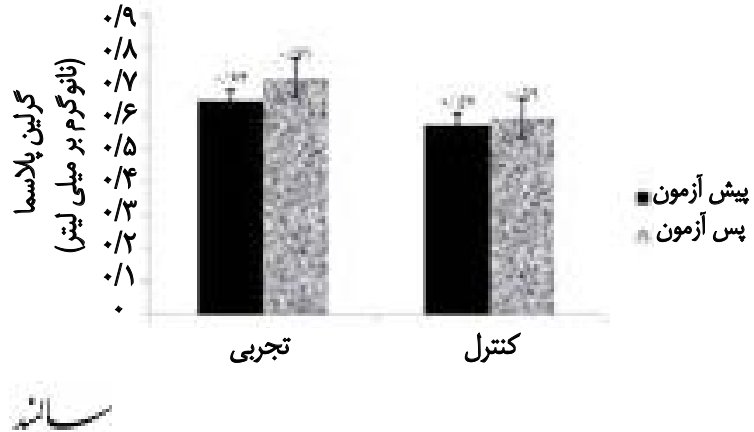

تصوير "ا. تغييرات سطح بيان رُن كرلين بلاسما در دو كروه آزمايش و كنترل

كروه شاهد معنى

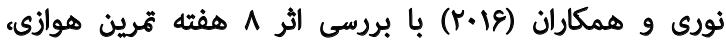

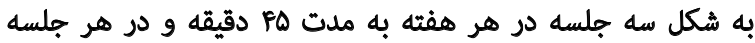

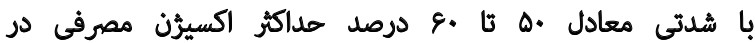

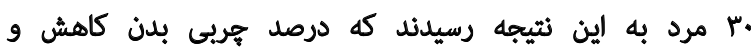

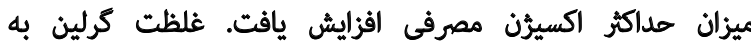

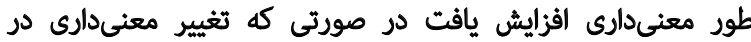

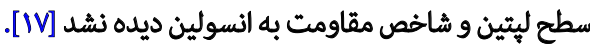

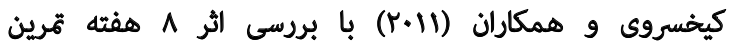

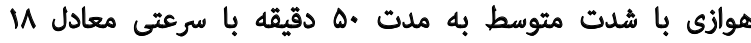

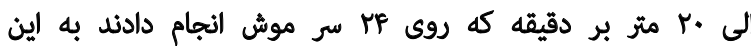

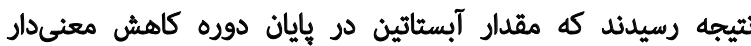

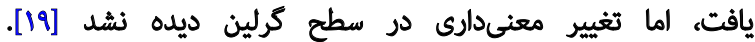

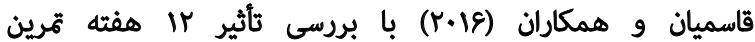

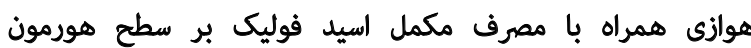

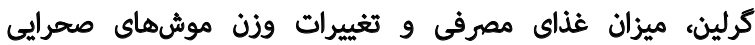

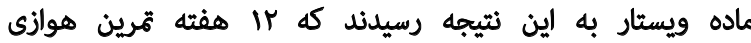

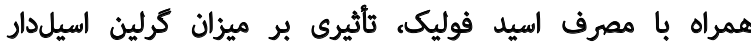

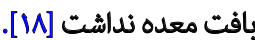

كزارشهاى تهقيقى مرتبط با بيان زٔن كرلين حاكى از آن

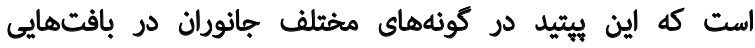

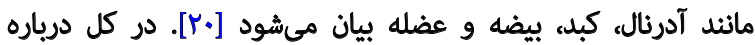

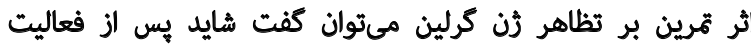

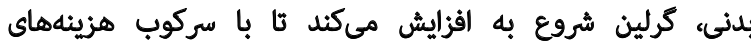

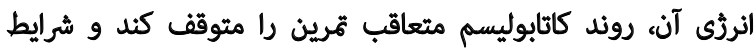

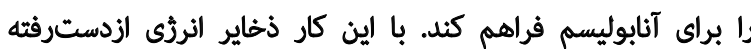

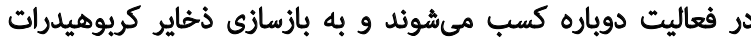
كمك هواهل شيد.

افزايش بيان زن كرلين را مىتوان با تعادل انرزى منفى درونى

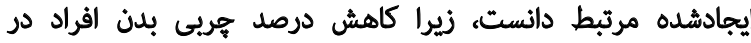

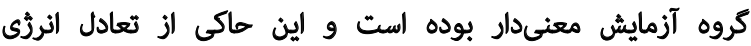

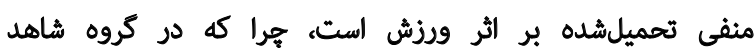


دانشكاه فردوسى مشهه با كد الم ال كرفته شده است.

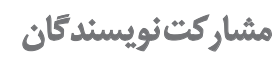

ثام نويسندكان در آمادهازى اين مقاله مشاركت داشتهاند.

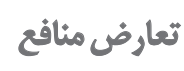

بنا بر اظهارنظر نويسندكان، تعارض منافعى وجود ندارد.
ثابت در بيهاران جاق است كه به دنبال جراحى عصب واك تنهائ

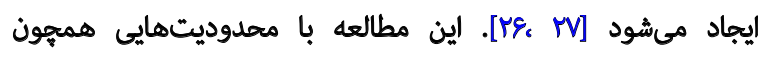

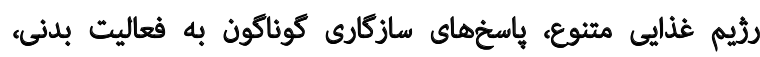

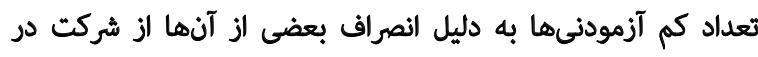

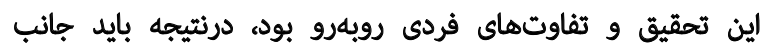

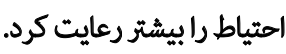

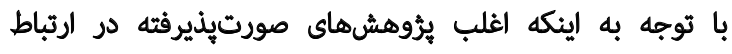

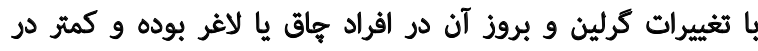

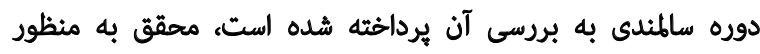

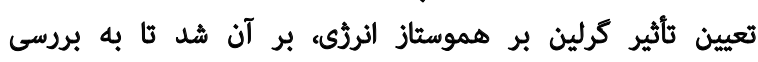

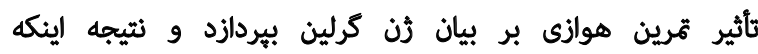

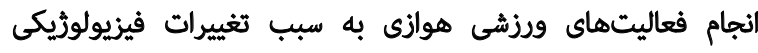

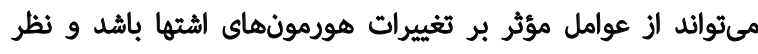

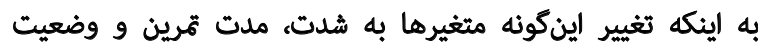

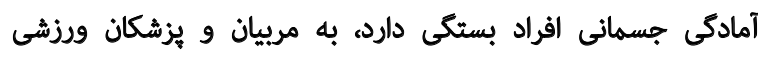

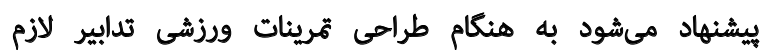
رابينديشند.

\section{نتيجلمَيرى نهمايى}

به طور كلى ^ هفته برنامه تمرين هوازى منجر به كاهش

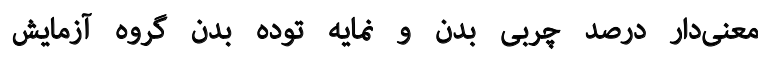

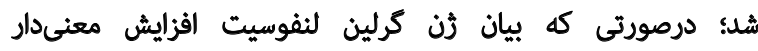

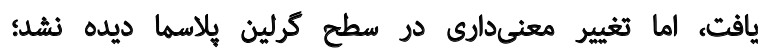

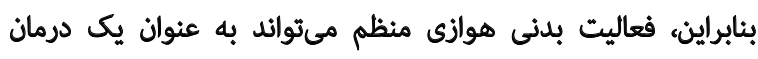

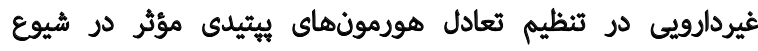

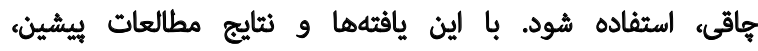

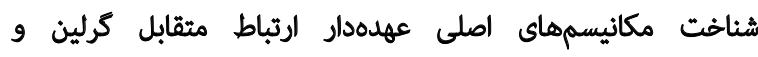

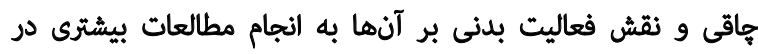

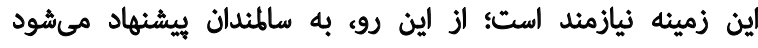

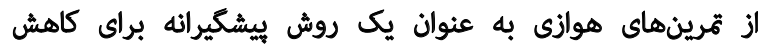
هاقى و بروز بيمارىهاي مرنبط با بآن استفاده كنند.

ملاحظات اخلاقي

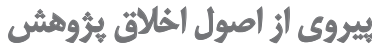

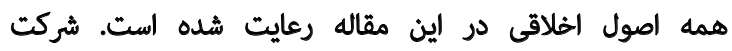

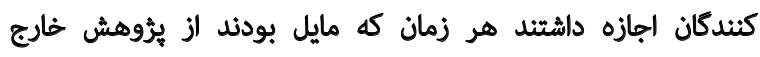

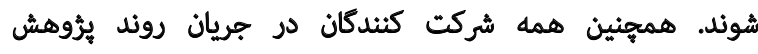

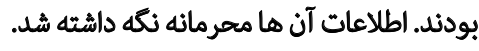

$$
\text { مامي مالى }
$$

اين مقاله از هاياننامه مقطع كارشناسى ارشد سيدمهدى

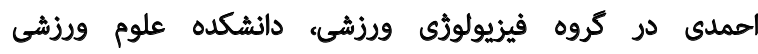




\section{References}

[1] Cheng MH, Bushnell D, Cannon DT, Kern M. Appetite regulation via exercise prior or subsequent to high-fat meal consumption. Appetite. 2009; 52(1):193-8. [DOI:10.1016/j.appet.2008.09.015]

[2] Issazadeh R. Effect of twelve weeks combined exercise (aerobicresistance) on plasma levels of ghrelin and obestatin in obese adolescens. Razi Journal of Medical Sciences. 2016; 22(141):91-103.

[3] Cummings DE. Ghrelin and the short-and long-term regulation of appetite and body weight. Physiology \& Behavior. 2006; 89(1):71-84. [DOI:10.1016/j.physbeh.2006.05.022]

[4] Cummings DE, Shannon MH. Roles for ghrelin in the regulation of appetite and body weight. Archives of Surgery. 2003; 138(4):389-96. [DOI:10.1001/archsurg.138.4.389]

[5] Ukkola O, Ravussin E, Jacobson P, Snyder EE, Chagnon M, Sjöström L, Bouchard C. Rapid communications: Mutations in the preproghrelin/ghrelin gene associated with obesity in humans. The Journal of Clinical Endocrinology \& Metabolism. 2001; 86(8):3996-9. [DOI:10.1210/jcem.86.8.7914] [PMID]

[6] Date Y, Nakazato M, Hashiguchi S, Dezaki K, Mondal MS, Hosoda $\mathrm{H}$, et al. Ghrelin is present in pancreatic a-cells of humans and rats and stimulates insulin secretion. Diabetes. 2002; 51(1):124-9. [DOI:10.2337/diabetes.51.1.124]

[7] Korbonits M, Goldstone AP, Gueorguiev M, Grossman AB. Ghrelin-a hormone with multiple functions. Frontiers in neuroendocrinology. 2004; 25(1):27-68.

[8] Woods SC, Benoit SC, Clegg DJ, Seeley RJ. Regulation of energy homeostasis by peripheral signals. Best Practice \& Research Clinical Endocrinology \& Metabolism. 2004; 18(4):497-515. [DOI:10.1016/j.beem.2004.08.004]

[9] Banks E, Lim L, Seubsman SA, Bain C, Sleigh A. Relationship of obesity to physical activity, domestic activities, and sedentary behaviours: cross-sectional findings from a national cohort of over 70,000 Thai adults. BMC Public Health. 2011; 11:762. [DOI:10.1186/1471-2458-11-762]

[10] Kraemer RR, Castracane VD. Exercise and humoral mediators of peripheral energy balance: Ghrelin and adiponectin. Experimental Biology and Medicine. 2007; (2):184-94.

[11] Olcucu B, Vatansever S, Koparan S, Mulazimoglu O, Çınar V. Effect of moderate intensity exercise on ghrelin and leptin hormones. Life Sciences. 2015; 5(3):1301-6.

[12] Azizi M. Serum leptin and ghrelin changes-induced aerobic training in healthy young females. International Journal of Collaborative Research on Internal Medicine \& Public Health. 2012; 4(6):1257-64

[13] Sternfeld B, Ainsworth BE, Quesenberry JR CP. Physical activity patterns in a diverse population of women. Preventive Medicine. 1999; 28(3):313-23. [DOI:10.1006/pmed.1998.0470]

[14] Kojima M,Hosoda H, Date Y, Nakazato M, Matsuo H, Kangawa $\mathrm{K}$. Ghrelin is a growth-hormone-releasing acylated peptide from stomach. Nature. 1999; 402(6762):656-60. [DOI:10.1038/45230]

[15] Aghapour A, Farzanegi P. Effect of six-week aerobic exercise on Chemerin and Resistin concentration in hypertensive postmenopausal women. Electronic Physician. 2013; 5(1):623-30.
[16] Fathi R, Ghanbari-Niaki A, Rahbarizadeh F, Hedayati MA Ghahramanloo E, et al. The effect of exercise on plasma acylated ghrelin concentrations and gastrocnemius muscle mRNA expression in male rats. Iranian Journal of Endocrinology and Metabolism. 2009; 10(5):519-26.

[17] Nuri R, Moghaddasi M, Darvishi H, Izadpanah A. Effect of aerobic exercise on leptin and ghrelin in patients with colorectal cancer. Journal of Cancer Research and Therapeutics. 2016 12(1):169-74. [DOI:10.4103/0973-1482.155982]

[18] Parvizi A, Ghasemnian A, Rahmani A. [Effect of 12 weeks aerobic exercise for along with folic acid supplementation on the levels of the ghrelin hormone amount of food intake and weight changes of female Wistar rats (Persian)]. Armaghan-e Danesh 2016; 21(8):746-56.

[19] Keikhosravi F, Shah HM, Daryanoush F, Hosseini SA, Noura $\mathrm{M}$, Hasanpour $\mathrm{G}$, et al. The effect of eight weeks moderate Intensity aerobic training on obestatin and ghrelin of obese male rats. Researcher in Sport Science Quarterly. 2011; 2(1):18-25.

[20] Ghelardoni S, Carnicelli V, Frascarelli S, Ronca-Testoni S, Zucchi R. Ghrelin tissue distribution: comparison between gene and protein expression. Journal of Endocrinological Investigation. 2006; 29(2):115-21. [DOI:10.1007/BF03344083]

[21] Morpurgo PS, Resnik M, Agosti F, Cappiello V, Sartorio A, Spada A. Ghrelin secretion in severely obese subjects before and after a 3-week integrated body mass reduction program. Journal of Endocrinological Investigation. 2003; 26(8):723-7.

[22] Chen JX, Zhao X, Yue GX, Wang ZF. Influence of acute and chronic treadmill exercise on rat plasma lactate and brain NPY LENK, DYN A 1-13. Cellular and Molecular Neurobiology. 2007; 27(1):1-10. [DOI:10.1007/s10571-006-9110-4]

[23] Hansen TK, Dall R, Hosoda H, Kojima M, Kangawa K, Christiansen JS, et al. Weight loss increases circulating levels of ghrelin in human obesity. Clinical Endocrinology. 2002; 56(2):203-6. [DOI:10.1046/j.0300-0664.2001.01456.x]

[24] Tschöp M, Weyer C, Tataranni PA, Devanarayan V, Ravus$\sin$ E, Heiman ML. Circulating ghrelin levels are decreased in human obesity. Diabetes. 2001; 50(4):707-9. [DOI:10.2337/diabetes.50.4.707]

[25] Tolle V, Kadem M, Bluet-Pajot MT, Frere D, Foulon C, Bossu $\mathrm{C}$, et al. Balance in ghrelin and leptin plasma levels in anorexia nervosa patients and constitutionally thin women. The Journal of Clinical Endocrinology \& Metabolism. 2003; 88(1):109-16. [DOI:10.1210/jc.2002-020645]

[26] Shiiya T, Nakazato M, Mizuta M, Date Y, Mondal MS, Tanaka $\mathrm{M}$, et al. Plasma ghrelin levels in lean and obese humans and the effect of glucose on ghrelin secretion. The Journal of Clinical Endocrinology \& Metabolism. 2002; 87(1):240-4. [DOI:10.1210/ jcem.87.1.8129]

[27] Andrews ZB, Liu ZW, Wallingford N, Erion DM, Borok E, Friedman JM, et al. UCP2 mediates ghrelin's action on NPY / AgRP neurons by lowering free radicals. Nature. 2008, 454(7206):846-51. [DOI:10.1038/nature07181] 
This Page Intentionally Left Blank 